TITLE:

\title{
Ultrasonic bandgaps in composite laminates: Experimental observation and Floquet-Bloch wave analysis
}

$\operatorname{AUTHOR}(\mathrm{S})$ :

Ishii, Yosuke; Biwa, Shiro

\section{CITATION:}

Ishii, Yosuke ... [et al]. Ultrasonic bandgaps in composite laminates: Experimental observation and Floquet-Bloch wave analysis. Applied Physics Letters 2018, 113(10): 101906.

ISSUE DATE:

2018-09-03

URL:

http://hdl.handle.net/2433/234643

\section{RIGHT:}

The following article appeared in Y. Ishii and S. Biwa: "Ultrasonic bandgaps in composite laminates: Experimental observation and Floquet-Bloch wave analysis", Applied Physics Letters, 113, 101906 (2018) and may be found at https://doi.org/10.1063/1.5046150.; The full-text file will be made open to the public on 03 September 2019 in accordance with publisher's 'Terms and Conditions for Self-Archiving'. 


\title{
Ultrasonic bandgaps in composite laminates: Experimental observation and Floquet-Bloch wave analysis
}

\author{
Yosuke Ishii ${ }^{\text {a) }}$ and Shiro Biwa ${ }^{\text {b) }}$ \\ Department of Aeronautics and Astronautics, Graduate School of Engineering, Kyoto University, Katsura, \\ Nishikyo-ku, Kyoto 615-8540, Japan
}

(Received 26 June 2018; accepted 20 August 2018; published online 6 September 2018)

\begin{abstract}
The ultrasonic bandgap characteristics of fiber-reinforced composite laminates are studied experimentally and theoretically. The transmission spectra of obliquely incident ultrasonic waves are obtained experimentally for 16-ply carbon/epoxy composite laminates having interleaf layers at interlaminar interfaces. Compared to the conventional composite laminates, these interleaved composite laminates exhibit clearer bandgaps in the transmission spectra and enable closer examination of their features. The transmission spectra for different incident angles are favorably reproduced by the theoretical plane wave transmission analysis based on the spring-type interlaminar interface model. The Floquet-Bloch wave analysis for the corresponding periodic structures is also employed to elucidate the ultrasonic bandgap mechanism in the composite laminates. Published by AIP Publishing. https://doi.org/10.1063/1.5046150
\end{abstract}

Ultrasonic waves offer an efficient means to characterize the mechanical properties of various composite materials. ${ }^{1,2}$ Fiber-reinforced polymer-matrix composites are typically manufactured in the form of laminates by stacking a number of reinforced layers (plies). As a result, thin resin regions remain at the interfaces between adjacent plies and act as scatterers to ultrasonic waves. Their effects on the overall wave propagation characteristics are negligible when the wavelength is sufficiently long. Based on this premise, several procedures have been proposed to identify the anisotropic (visco)elastic constants of laminates ${ }^{3-7}$ as well as those of constituting plies. ${ }^{8,9}$ On the other hand, when the wavelength is comparable with the double ply thickness, the wave scattered at interlaminar interfaces can interfere constructively to prohibit the wave transmission through the laminate, which corresponds to the bandgap in periodically layered systems. In the temporal reflection waveforms, this can be observed as persistent echoes from the interlaminar interfaces. $^{10}$

In assessing the structural integrity of composite laminate structures, the quality of interlaminar interfaces is of equal or higher importance compared to the mechanical properties of plies themselves. In recent investigations, a method has been proposed ${ }^{11-15}$ to evaluate the equivalent stiffnesses of interlaminar interfaces of composite laminates based on the reflection or transmission spectra of ultrasonic waves in the frequency range where the wavelength is comparable to the double ply thickness. Furthermore, it has been shown that the porosity content in the laminate can be evaluated from the characteristics of interlaminar interface echoes in the frequency range of bandgap. ${ }^{10}$ These studies highlight the importance of gaining fundamental understanding of the ultrasonic bandgap features of composite laminates in order

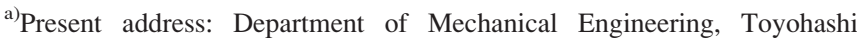
University of Technology, 1-1 Hibarigaoka, Tempaku-cho, Toyohashi 441-8580, Japan.

b) Author to whom correspondence should be addressed: biwa@kuaero.kyoto-u. ac.jp.
}

to utilize them for nondestructive materials characterization. In the above studies, ${ }^{10-15}$ however, conventional carbon fiber-reinforced composite laminates were used for experimental observations, whose interlaminar resin regions were so thin (only a few microns) that the interlaminar scattering was very weak. As a consequence, it was difficult to observe the bandgaps in the reflection/transmission spectra in a clear manner, which hindered in-depth examination of the bandgap mechanism.

In this letter, the experimental observation of ultrasonic bandgaps in the oblique transmission characteristics is demonstrated for another type of composite laminates which have thicker interlaminar resin regions. These composite laminates are shown to have ultrasonic bandgaps in a more enhanced manner, which is of advantage in performing the comparison with the theoretical model and elucidating the physical phenomena involved in more details. Moreover, the examination of this type of composite laminates is of practical importance since these composites are now widely used for aircraft structures, known as interlaminar-toughened composite laminates. ${ }^{16}$ The bandgap behavior will also be discussed theoretically based on the spring-type interlaminar interface model and the Floquet-Bloch wave theory. ${ }^{17}$ The Floquet-Bloch theory was employed in Refs. 14 and 15 to examine the condition for bandgap formation in composite laminates. In this letter, the theory will be used to further explore the physical mechanism of the bandgaps observed experimentally, i.e., to show the number of propagating Floquet-Bloch modes for a given frequency and incident angle, and to clarify the Floquet-Bloch mode(s) which are responsible for each ultrasonic bandgap.

Two kinds of carbon/epoxy composite laminates consisting of 16 plies fabricated using Toray T800S/3900-2B prepregs were used as the specimens, i.e., a unidirectional (UD) laminate with the stacking sequence $[0]_{16}$ and a quasiisotropic (QI) laminate with $[-45 / 0 /+45 / 90]_{2 S}$. These laminates have interleaf layers with thickness of 20-30 $\mu \mathrm{m}$ made of heterogeneous resin containing fine thermoplastic 
particles: ${ }^{18}$ the presence of such layers contributes to improved interlaminar fracture toughness and resistance to the onset of delaminations. ${ }^{19,20}$ The total thickness of these laminates is around $3 \mathrm{~mm}$. These specimens were referred to as interleaved laminates in this letter to distinguish them from conventional composite laminates.

Following the procedure outlined in Ref. 15, the through-transmission measurement was performed for each specimen immersed in water with various incident directions determined by the angle of incidence $\theta$ and the angle of the plane of incidence $\varphi$ (see the inset in Fig. 1). Here, the angle $\varphi$ is defined in such a manner that $\varphi=0^{\circ}$ corresponds to the plane whose normal coincides with the fiber direction of the $0^{\circ}$ plies. The ultrasonic wave was emitted by exciting a $10 \mathrm{MHz}$ piezoelectric transducer (diameter $0.5 \mathrm{in}$.) by a wideband spike signal, and the transmitted wave was received by another $10 \mathrm{MHz}$ transducer (diameter 1 in.) on the other side of the specimen. The transmission waveforms measured for the interleaved UD laminate are shown in Fig. 1 for some representative incident directions, together with the reference signal measured in water with the same transducer layout but in the absence of the specimen.

The transmission waveforms in Fig. 1 were Fourier transformed with a rectangular window ranging from 32 to $42 \mu \mathrm{s}$, and the resulting power spectra were normalized by that of reference signal to obtain the energy transmission spectra as shown in Fig. 2. In Fig. 2, the transmission coefficient shows clear dips at some frequencies as indicated by arrows. The meaning of the symbols such as "L" and "T1T2" attached to the dips will be explained later. These dips correspond to the stop bands originating from the constructive interference of scattered waves from the interlaminar interfaces ${ }^{14}$ and their location is clearly influenced by the incident direction. The results for the conventional 16-ply UD laminate of the similar thickness, fabricated using Toho Tenax UTS50/\#135 prepregs, are also shown in Fig. 2 for comparison. It is noted that the interleaved laminate exhibits much deeper stop bands. The transmission spectra for the interleaved composite laminate are shown in Fig. 3(a) for the

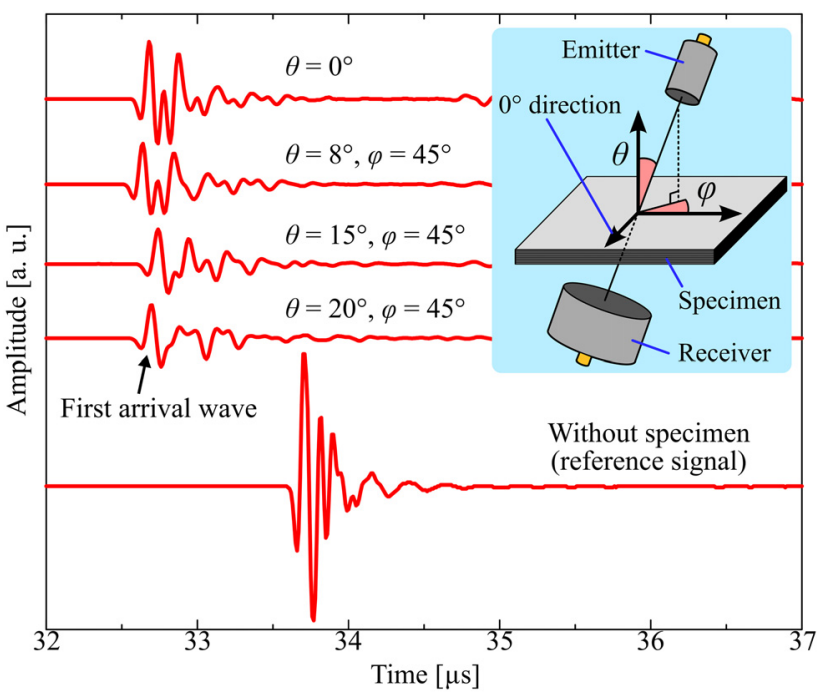

FIG. 1. Transmission waveforms measured for the interleaved UD specimen together with the reference signal.

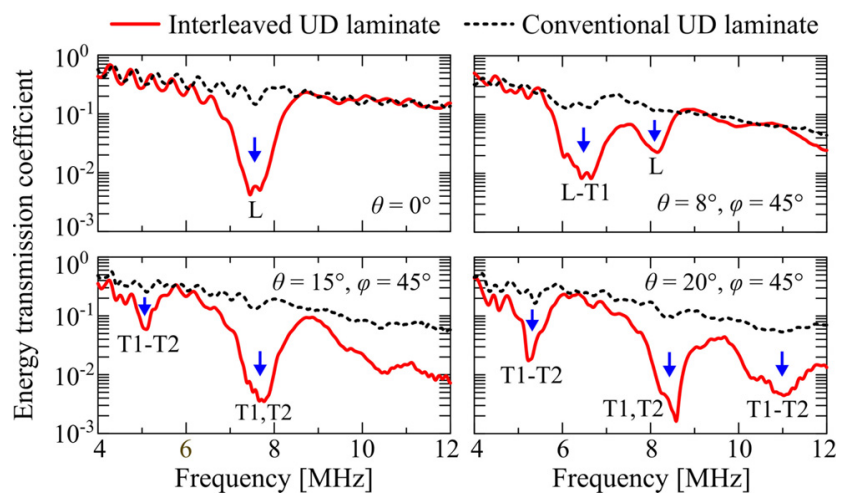

FIG. 2. Energy transmission spectra for the waveforms in Fig. 1. The bandgaps are indicated by arrows and the symbols indicating the Floquet mode(s) which are responsible.

range $0^{\circ}<\theta<50^{\circ}$ and for $\varphi=0^{\circ}, 45^{\circ}$, and $90^{\circ}$. In the plane of frequency and incident angle, it is seen that the transmission coefficient becomes vanishingly small in some curved strip-shaped zones.

In order to interpret these results theoretically, the transmission coefficient was calculated for oblique incidence of plane monochromatic longitudinal wave on the 16-ply laminate structure immersed in water using the stiffness-matrix method. ${ }^{21}$ The plies were modeled as a transversely isotropic linear viscoelastic solid, and the interlaminar resin regions as a mass-less spring-type interface of finite interfacial stiffnesses in the normal and tangential directions $\left(K_{\mathrm{N}}\right.$ and $K_{\mathrm{T}}$, respectively). ${ }^{14,15}$ The ply and interface parameters were determined so that the theoretical distribution of energy transmission coefficient in the plane of frequency and incident angle fits best to the experimental result using the same
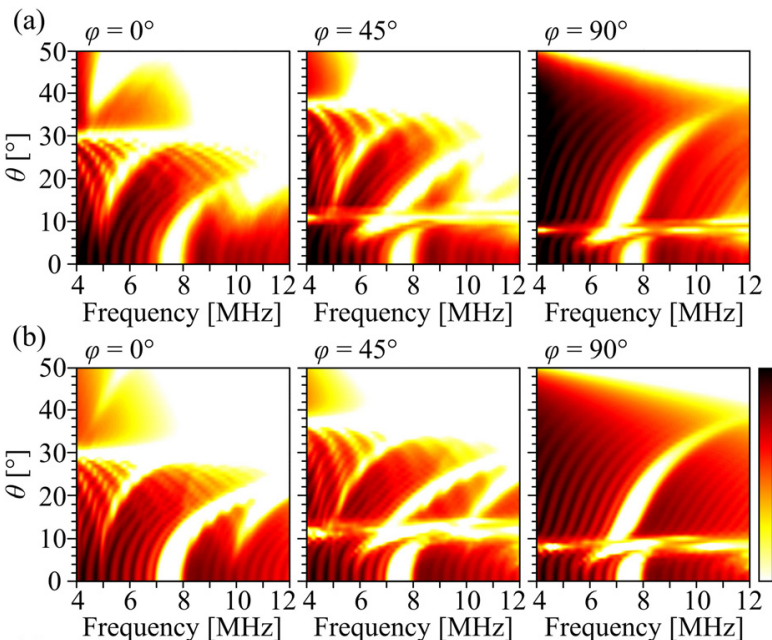

$\varphi=45^{\circ}$
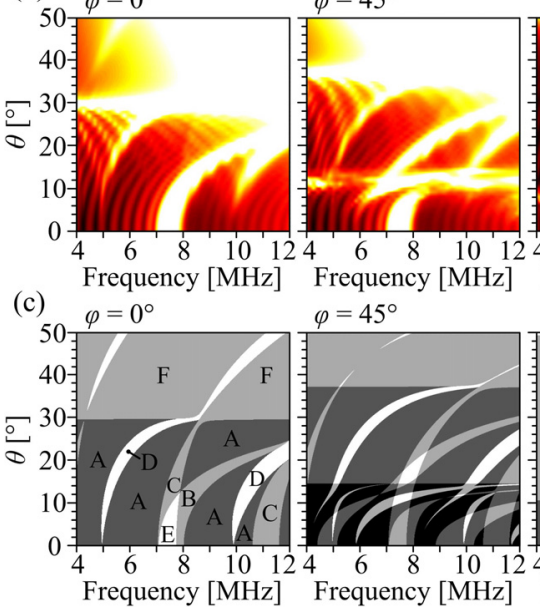

$\varphi=90^{\circ}$
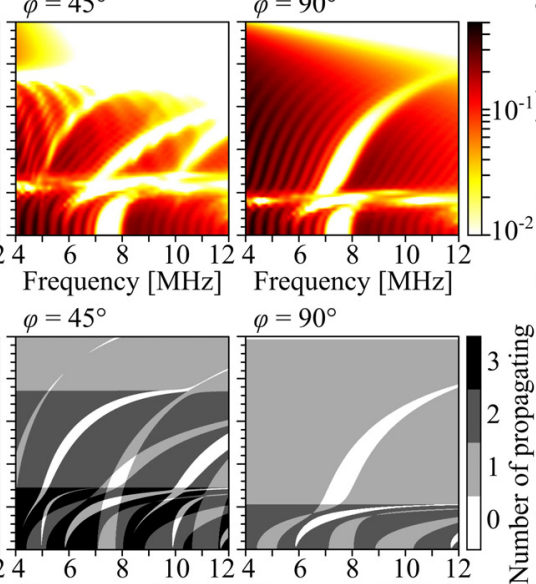

$\varphi=90^{\circ}$

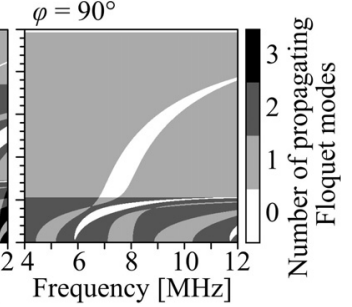

FIG. 3. Energy transmission spectra of interleaved UD laminate for different incident angles and for three planes of incidence, (a) experiment, (b) theoretical analysis, with (c) the number of propagating Floquet modes in the infinite periodic layered structure. 
optimization method as described in Ref. 15 (the ply density and the thickness were obtained from measurements). With these parameters shown in Table I, the theoretical transmission spectra in Fig. 3(b) match closely with the experimental results in Fig. 3(a), with the presence of low-transmission zones at the similar frequencies and incident angles. The identified ply complex moduli are representative of carbon/ epoxy composites. ${ }^{22}$ The interlaminar interfacial stiffnesses in Table I also appear reasonable since the approximate estimation ${ }^{11,23}$ of equivalent interfacial stiffnesses of a thin layer of uniform thickness gives $K_{\mathrm{N}}=0.1-0.6 \mathrm{GPa} / \mu \mathrm{m}$ and $K_{\mathrm{T}}=0.04-0.1 \mathrm{GPa} / \mu \mathrm{m}$ for the range of Young's modulus $E_{\mathrm{r}}=3.5-4.8 \mathrm{GPa}$ and Poisson's ratio $\nu_{\mathrm{r}}=0.24-0.42$ estimated for the interleaf layer of this composite ${ }^{18}$ and for the layer thickness $20-30 \mu \mathrm{m}$. It is also noted that these interfacial stiffnesses are much smaller than those obtained for conventional composite laminates $\left(K_{\mathrm{N}}=3.0 \mathrm{GPa} / \mu \mathrm{m}\right.$ and $\left.K_{\mathrm{T}}=0.8 \mathrm{GPa} / \mu \mathrm{m}\right),{ }^{14,15}$ which accounts for the much clearer bandgaps observed for the present specimen.

In the previous studies, ${ }^{14,15}$ the low-transmission zones in the transmission spectra of composite laminates were closely related to the bandgaps of Floquet-Bloch waves (simply referred to as Floquet waves hereafter) in the corresponding infinitely extended periodic laminate structures. For a given frequency, there are three pairs of Floquet wavenumbers of opposite signs in the first Brillouin zone which are complex in general. Each of three Floquet modes is dominated either by the longitudinal (L), fast transverse (T1), or slow transverse (T2) bulk wave in each ply. In order to examine the connection of the Floquet wave features to Fig. 3 (b), it is useful to examine the number of real Floquet wavenumbers (the number of propagating Floquet modes) which have the same horizontal component with the wavenumber of the longitudinal wave in water, as depicted in Fig. 3(c) as a function of frequency and incident angle. It has been shown that a bandgap is formed when the following condition is satisfied ${ }^{14}$

$$
\operatorname{Re}\left[\zeta_{\alpha}\right] h_{\mathrm{u}}+\operatorname{Re}\left[\zeta_{\beta}\right] h_{\mathrm{u}}=2 n \pi, \quad(\alpha, \beta=1,2,3),
$$

where each of $\zeta_{\alpha}$ and $\zeta_{\beta}$ is one of the three Floquet wavenumbers, $h_{\mathrm{u}}$ is the spatial period of the layered structure, and

TABLE I. Material properties used for the stiffness-matrix calculation.

\begin{tabular}{lcc}
\hline \hline & UD & QI \\
\hline Ply density $\left(\mathrm{kg} / \mathrm{m}^{3}\right)$ & $1.55 \times 10^{3}$ & $1.55 \times 10^{3}$ \\
Ply thickness $(\mathrm{mm})$ & 0.19 & 0.19 \\
Ply complex moduli $(\mathrm{GPa})^{\mathrm{a}}$ & $103-3.3 \mathrm{i}$ & $122-3.6 \mathrm{i}$ \\
$C_{11}$ & $5.0-0.23 \mathrm{i}$ & $5.9-0.21 \mathrm{i}$ \\
$C_{13}$ & $14.8-0.28 \mathrm{i}$ & $14.9-0.26 \mathrm{i}$ \\
$C_{33}$ & $3.4-0.12 \mathrm{i}$ & $3.4-0.14 \mathrm{i}$ \\
$C_{44}$ & $6.1-0.20 \mathrm{i}$ & $6.0-0.26 \mathrm{i}$ \\
$C_{66}$ & \multicolumn{2}{c}{0.59} \\
Interlaminar interfacial stiffnesses $(\mathrm{GPa} / \mu \mathrm{m})$ & 0.57 & 0.14 \\
$\quad$ Normal component $K_{\mathrm{N}}$ & 0.18 & \multicolumn{2}{c}{$1.0 \times 10^{3}$} \\
$\quad$ Tangential component $K_{\mathrm{T}}$ & \multicolumn{2}{c}{1.5} \\
Density of water $\left(\mathrm{kg} / \mathrm{m}^{3}\right)$ & \multicolumn{2}{c}{} \\
Wave speed in water $(\mathrm{km} / \mathrm{s})$ & \multicolumn{2}{c}{} \\
\hline \hline
\end{tabular}

${ }^{\mathrm{a}}$ Transverse isotropy with the fiber direction set as the $x_{1}$ axis is assumed. $n$ stands for an integer. If $\zeta_{\alpha}$ and $\zeta_{\beta}$ are the wavenumbers for the same Floquet mode, the number of propagating Floquet modes reduces by one, and if they are for different Floquet modes, this number reduces by two.

The appearance of bandgaps is now examined for the case of $\varphi=0^{\circ}$ as an illustrative example. The dispersion relation of Floquet waves for $\theta=8^{\circ}$ and $\varphi=0^{\circ}$ is shown in Fig. 4. In Figs. 3(c) and 4, different regions are marked as A to $\mathrm{F}$. In region $\mathrm{A}$, two Floquet modes can propagate in the structure, i.e., one dominated by the L-wave and the other dominated by the T2-wave in the plies. In the regions B, C, and D, Eq. (1) is satisfied by the L-dominated Floquet mode, by the T2-dominated Floquet mode, and by the combination of the L-dominated and T2-dominated Floquet modes, respectively, and the wavenumber of the involved Floquet mode(s) becomes non-real. As a result, only the T2dominated (L-dominated) Floquet mode can propagate in region $\mathrm{B}(\mathrm{C})$, while no Floquet modes can propagate in region $\mathrm{D}$. In region E, Eq. (1) is met by both the L- and T2dominated modes, so both modes are evanescent there. In region F, the L-dominated Floquet mode becomes evanescent as the incident angle exceeds the critical angle (about $29.7^{\circ}$ ) for that mode, so only the T2-dominated Floquet mode can propagate in the laminate. Such interpretation is possible for other cases in Fig. 3(c) by examining the corresponding dispersion relation of Floquet modes. It is noted that for $\varphi=45^{\circ}$, there are three relevant Floquet modes dominated by the L, T1, and T2 waves, respectively, and the fulfillment of Eq. (1) is achieved with richer combination of these Floquet modes. In Fig. 2, the Floquet mode(s) involved in each bandgap are shown by the symbols such as "L" and "T1-T2": "L" means that the condition of Eq. (1) is satisfied by the L-dominated Floquet mode, "T1-T2" means that it is satisfied by the combination of the T1-dominated and T2dominated Floquet modes, "T1, T2" means that it is satisfied simultaneously by these two modes, and other symbols likewise.

The above characteristics of Floquet waves in the infinite layered structure explain many of the features of lowtransmission zones for the finite laminate in Figs. 3(a) and 3 (b). Below the critical angle $\left(29^{\circ}\right)$, the longitudinal wave in

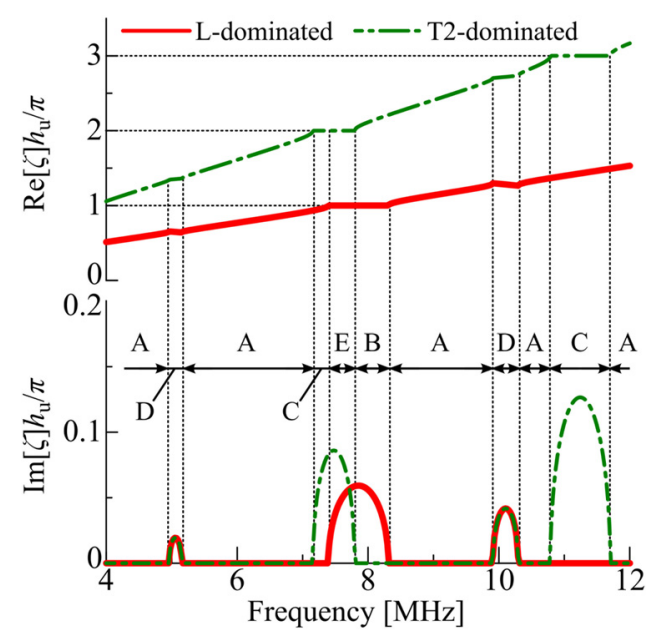

FIG. 4. Dispersion relation of Floquet waves for the periodic structure of interleaved UD composite when $\theta=8^{\circ}$ and $\varphi=0^{\circ}$. 
water is most efficiently transmitted through the laminate by coupling with the longitudinal (or quasi-longitudinal) wave in the plies. Therefore, the low-transmission zones match closely with the regions of Fig. 3(c), where the L-dominated Floquet mode is involved in the bandgap, i.e., regions B, D, and E. In Figs. 3(a) and 3(b), there are some additional features due to the finite thickness of the laminate. In particular, the transmission coefficient of the finite laminate oscillates as a function of the frequency, thus exhibiting stripe patterns, outside of the bandgaps. Furthermore, the transmission coefficient in Figs. 3(a) and 3(b) becomes vanishingly small at the critical angles of Floquet mode, ${ }^{24}$ i.e., approximately $\theta=29.7^{\circ}$ for $\varphi=0^{\circ}, \theta=14.6^{\circ}$ and $37.3^{\circ}$ for $\varphi=45^{\circ}$, and $\theta=10.6^{\circ}$ and $49.4^{\circ}$ for $\varphi=90^{\circ}$.

The corresponding results for the QI laminate are shown in Fig. 5. The theoretical results in Fig. 5(b) were calculated with the ply and interfacial parameters as shown in Table I, which were also determined by best fitting the theoretical model to the experimental results. Due to the multidirectional nature of this laminate, the dependence of the transmission coefficient on the frequency and the incident angle is far more complicated in Fig. 5 compared to Fig. 3. For this laminate, the Floquet theory can be applied by taking four consecutive plies with the interlaminar interfaces as the unit cell. ${ }^{15}$ In this theory, the three cases of $\varphi=0^{\circ}, 45^{\circ}$, and $90^{\circ}$ correspond to the same Floquet wave characteristics as shown in Figs. 5(c) and 5(d). For low incident angles up to about $10^{\circ}$, the observed low-transmission zones can be interpreted by the Floquet wave features in the manner similar to the UD laminate. For higher incident angles, however, a finite-thickness effect can be observed that the three cases show different transmission spectra depending on the relative orientation of the plane of incidence to the fiber direction of
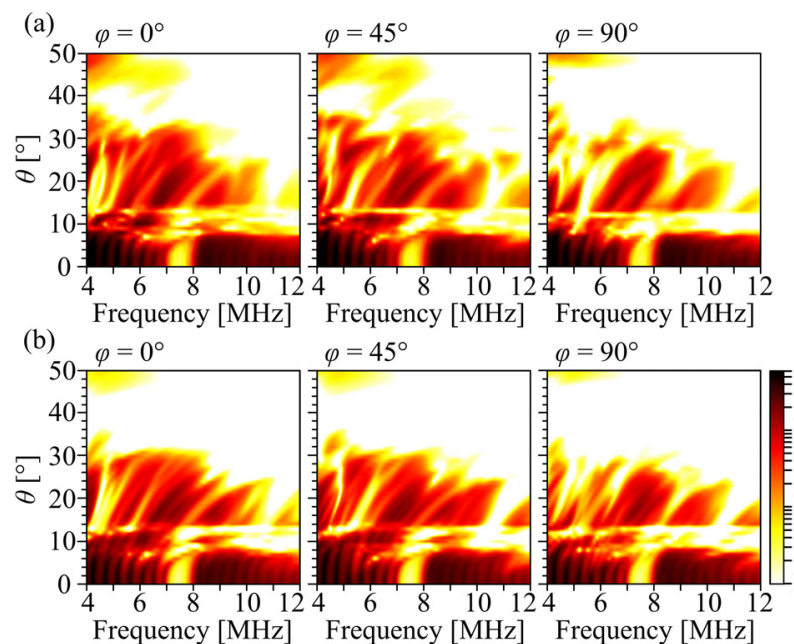

$\varphi=45^{\circ}$

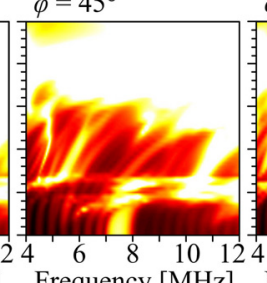

$\varphi=90^{\circ}$
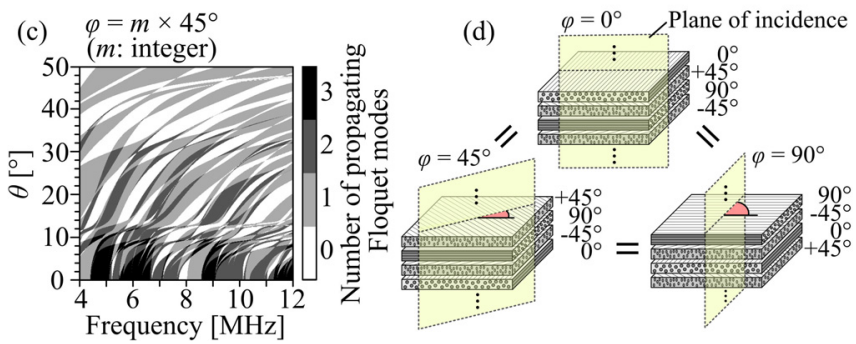

FIG. 5. The same as in Fig. 3, but for the interleaved QI laminate. the surface ply. It is notable that the measured transmission spectra in Fig. 5(a) are quite reasonably reproduced by the theoretical modeling for this laminate, too, with similar ply and interfacial parameters to those of the UD laminate as shown in Table I.

In summary, the ultrasonic bandgap features have been demonstrated experimentally for carbon/epoxy composite laminates with interleaf layers. Based on the Floquet wave analysis, the formation mechanism of the bandgaps involving different Floquet modes has been elucidated, and the low-transmission behavior experimentally observed in some ranges of frequency and incident angle has been interpreted theoretically. Furthermore, it has been demonstrated that the ply complex moduli and the interlaminar interfacial stiffnesses can be evaluated by fitting the theoretical model to the experimental transmission spectra, owing to the rich information contained in the bandgap features. Rigorously speaking, the spring-type interface model used in the theoretical analysis is valid when the interlaminar resin regions are sufficiently thin compared to the wavelength in question. This is certainly the case for conventional composite laminates with a few micron-thick interlaminar resin regions. For interleaved laminates, however, the interleaf layers with thickness of typically $20-30 \mu \mathrm{m}$ may not be negligible compared to the shear wavelength $(0.1-0.3 \mathrm{~mm}$ for the present frequency range), which makes the premise of the springtype interface model somewhat questionable. Alternative approach is to model the interleaf layers as homogeneous layers of finite thickness with finite mass density and elastic properties. Such an approach can lead to the evaluation of the elastic constants of interleaf layers of composite laminates based on the transmission spectra with bandgap features, and will constitute a subject of our subsequent investigation.

This work was partly supported by JSPS KAKENHI Grant Nos. JP25-1754 and JP15K13833. The authors are grateful to the Aerospace Company, Kawasaki Heavy Industries, Ltd., for providing the composite laminate samples used in this study.

${ }^{1}$ R. A. Kline, Nondestructive Characterization of Composite Media (Technomic Publishing Co, 1992).

${ }^{2}$ S. I. Rokhlin, D. E. Chimenti, and P. B. Nagy, Physical Ultrasonics of Composites (Oxford University Press, 2011).

${ }^{3}$ M. F. Markham, Composites 1, 145 (1970).

${ }^{4}$ S. I. Rokhlin and W. Wang, J. Acoust. Soc. Am. 91, 3303 (1992).

${ }^{5}$ B. Hosten, M. Deschamps, and B. R. Tittmann, J. Acoust. Soc. Am. 82, 1763 (1987).

${ }^{6}$ S. Baudouin and B. Hosten, Ultrasonics 34, 379 (1996).

${ }^{7}$ M. Castaings, B. Hosten, and T. Kundu, NDT\&E Int. 33, 377 (2000).

${ }^{8}$ Y. C. Chu and S. I. Rokhlin, J. Acoust. Soc. Am. 96, 342 (1994).

${ }^{9}$ L. Wang and S. I. Rokhlin, J. Acoust. Soc. Am. 112, 916 (2002).

${ }^{10}$ Y. Ishii, S. Biwa, and A. Kuraishi, Compos. Struct. 152, 645 (2016).

${ }^{11}$ Y. Ishii and S. Biwa, J. Appl. Phys. 111, 084907 (2012).

${ }^{12}$ Y. Ishii and S. Biwa, J. Acoust. Soc. Am. 136, 183 (2014).

${ }^{13}$ Y. Ishii and S. Biwa, Phys. Procedia 70, 372 (2015).

${ }^{14}$ Y. Ishii and S. Biwa, J. Acoust. Soc. Am. 138, 2800 (2015).

${ }^{15}$ Y. Ishii and S. Biwa, J. Acoust. Soc. Am. 141, 1099 (2017).

${ }^{16}$ The Society of Fiber Science and Technology Japan, High-Performance and Specialty Fibers : Concepts, Technology and Modern Applications of Man-Made Fibers for the Future (Springer, Tokyo, 2016).

${ }^{17}$ A. M. B. Braga and G. Herrmann, J. Acoust. Soc. Am. 91, 1211 (1992).

${ }^{18}$ Y. Okabe and N. Takeda, J. Compos. Mater. 36, 757 (2002). 
${ }^{19}$ M. Hojo, S. Matsuda, M. Tanaka, S. Ochiai, and A. Murakami, Compos. Sci. Technol. 66, 665 (2006).

${ }^{20}$ N. Sato, M. Hojo, and M. Nishikawa, Composites, Part A 68, 202 (2015).
${ }^{21}$ L. Wang and S. I. Rokhlin, Ultrasonics 39, 413 (2001).

${ }^{22}$ B. Hosten and M. Castaings, J. Acoust. Soc. Am. 94, 1488 (1993).

${ }^{23}$ S. I. Rokhlin and Y. J. Wang, J. Acoust. Soc. Am. 89, 503 (1991).

${ }^{24}$ L. Wang and S. I. Rokhlin, J. Acoust. Soc. Am. 114, 2582 (2003). 